\title{
EVALUATION OF BLOOD PRESSURE VARIATIONS DURING THE ADMINISTRATION OF INTRAVASCULAR CONTRAST MEDIA IN CECT ABDOMEN
}

\author{
ARATHY MARY JOHN, SUSHIL YADAV* \\ Department of Medical Imaging Technology, SOAHS, Manipal Academy of Higher Education, Manipal - 576 104, Karnataka, India. \\ Email: sushil.yadav@manipal.edu
}

Received: 14 February 2018, Revised and Accepted: 14 March 2018

\section{ABSTRACT}

Objective: Hemodynamic changes may occur with the rapid intravenous injection of contrast media due to the osmolality of contrast media. This study is aimed to evaluate the blood pressure variation during the administration of intravascular contrast media in contrast-enhanced computed tomography (CECT) of the abdomen.

Methods: The study was performed in 146 subjects, who underwent abdomen CT scan with 64-slice Philips Brilliance CT scanner in the department of radio-diagnosis and imaging. Using convenience sampling technique, 73 subjects who were referred for CECT abdomen scan and another 73 subjects who were referred for non-CECT (NCECT) abdomen scan were included in the study. Among the CECT group, intravascular contrast media was injected into the patient during the scan. Both systolic and diastolic blood pressures were recorded just before and immediately after the scan in CECT and NCECT groups, by two readers independently. Mean systolic and diastolic blood pressure for both groups was calculated with standard deviation. The data were analyzed using repeated measures of ANOVA.

Results: Both systolic and diastolic blood pressures increased with the injection of contrast media among CECT scan group. No significant changes in systolic and diastolic blood pressure were found before and after the scan of NCECT group.

Conclusion: An increase in both systolic and diastolic blood pressures is associated with the intravascular administration of non-ionic low-osmolar contrast media.

Keywords: Abdomen scan, Computed tomography, Contrast enhanced, Diastolic blood pressure, Intravenous administration, Low-osmolar contrast media, Non-contrast enhanced, Non-ionic contrast media, Systolic blood pressure.

(c) 2018 The Authors. Published by Innovare Academic Sciences Pvt Ltd. This is an open access article under the CC BY license (http://creativecommons. org/licenses/by/4. 0/) DOI: http://dx.doi.org/10.22159/ajpcr.2018.v11i6.25284

\section{INTRODUCTION}

The practice of intravenous administration of contrast media during computed tomography (CT) scan has been continuously updated with the gradual development of CT technology [1]. There will be potential side effects or adverse reactions with every medication [2]. Same way administration of iodinated contrast media can be followed by some adverse reactions based on the characteristics of radiopaque contrast media, and its occurrence ranges from $0.2 \%$ to $12.7 \%$ of contrast injections [3]. Significant hemodynamic changes with a rapid intravenous injection of contrast media have been observed which was related to the osmolality of contrast media [4]. The hypertensive crisis was reported with administration of intravascular contrast media in CT examination of patients with pheochromocytoma during the 1960s and 1970s [5, 6]. These procedures used high-osmolar ionic contrast media which is more prone to induce adverse reactions compared to non-ionic low-osmolar contrast media [7]. However, with the vascular administration of non-ionic contrast media during an interventional procedure, a decrease in blood pressure was reported [8].

Nowadays, most of the contrast-enhanced CT (CECT) scans are performed with non-ionic low-osmolar contrast media [9]. Hypertension is considered as one of the leading causes of cardiovascular disease which accounts for a large proportion of death and disability [10]. Since hypertensive crisis is reported with the administration of high-osmolar contrast media, administration of low-osmolar contrast media also needs to be evaluated for the occurrence of blood pressure variation. In this study, we aim to study the effect of intravascular administration of low-osmolar non-ionic contrast media on blood pressure during CECT examination of the abdomen.

\section{METHODS}

A prospective cross-sectional study was conducted on approval of the Institutional Research Committee, SOAHS, and Ethical Committee, Kasturba Hospital. Using convenience sampling technique, 73 subjects who were referred for CECT abdomen scan and another 73 subjects who were referred for non-CECT (NCECT) abdomen scan during the study period from March 2015 to March 2016 were included in the study. The patients with a history of hypertension and patients with delay scanning after contrast injection were excluded from the study. All the scans were performed using Philips, 64-slice Brilliance CT at the Department of Radio-diagnosis and Imaging, Kasturba Hospital, Manipal.

Intravenous non-ionic low-osmolar contrast media was injected at a rate of $4 \mathrm{ml} / \mathrm{s}$ among the subjects who underwent for CECT abdomen scan. Amount of contrast injected was based on the weight of the subject at a rate of $2 \mathrm{ml} / \mathrm{kg}$, with a maximum amount of $80 \mathrm{ml}$. Systolic and diastolic blood pressure was measured just before the injection of contrast media and immediately after the portal venous phase of abdomen scan, using a sphygmomanometer, with the patient lying on the examination table. Blood pressure was measured twice with two independent readers who are blind to the injection of contrast media to avoid the reader bias. In case of NCECT patients, blood pressure was measured just before the scan and immediately after the scan with the patient lying on the examination table, by two readers separately. The gap between two measurements was within 5 min. Mean systolic and diastolic blood pressure for both groups was calculated with standard deviation.

Mean systolic and diastolic blood pressure before and after the injection of contrast media was compared to find out the effect of intravascular 
contrast media on blood pressure among CECT group. Mean systolic and diastolic blood pressure before and after the NCECT scan group was compared to find out the blood pressure variations that can arise due to anxiety during the scan. The data collected during this study were analyzed using repeated measures of ANOVA with 95\% confidence interval. All statistical analyses were done using SPSS 16.0

\section{RESULTS}

The mean of systolic blood pressure is summarized in Table 1. Among the patients who underwent for CECT scan, the mean systolic blood pressure after the administration of intravenous contrast media was raised to $128.08 \pm 6.489 \mathrm{mmHg}$ from $116.64 \pm 6.349 \mathrm{mmHg}$. However, among NCECT group, minute increase in systolic blood pressure was noted between pre- and post-scans from $119.59 \pm 5.188 \mathrm{mmHg}$ to $120.14 \pm 5.832 \mathrm{mmHg}$

While comparing the systolic blood pressure after the scan in CECT and NCECT abdomen, a statistically significant increase in systolic blood pressure was observed with contrast injection $(\mathrm{p}<0.050)$

The mean of diastolic blood pressure is summarized in Table 2 . The mean diastolic blood pressure after the administration of intravenous contrast agent in CECT was found to be increased from $77.33 \pm 6.351 \mathrm{mmHg}$ to $87.19 \pm 5.95 \mathrm{mmHg}$ (Fig. 2). Among NCECT group, the mean diastolic blood pressure before and after the scan was $80.41 \pm 6.495 \mathrm{mmHg}$ and $81.51 \pm 7.102 \mathrm{mmHg}$, respectively.

While comparing the diastolic blood pressure after the scan in CECT and NCECT abdomen, a statistically significant increase in diastolic blood pressure was observed with contrast injection $(\mathrm{p}<0.050)$.

\section{DISCUSSION}

In our study, we analyzed the variation in systolic and diastolic blood pressure before and after injecting the contrast media in CECT abdomen scan. We also analyzed the same in NCECT abdomen scan to comment on the blood pressure variations that can arise due to anxiety during the scan. In CECT group, an increase in both systolic and diastolic blood pressures was observed with intravascular administration of lowosmolar contrast media. Among NCECT group, a very slight increase in systolic and diastolic blood pressure was observed, but it was not clinically significant.

A study which supports our finding was conducted by Sachiko et al. in which hypertensive crisis was reported with the injection of non-ionic low-osmolar contrast media during CT examination [9]. They suspected an additional release of catecholamine triggered by the injection of contrast media which lead to the hypertensive crisis. Catecholamine level after the injection of contrast media was not done in our study. However, Bald et al. found that intravenous low-osmolar contrast administration during CT did not induce catecholamine release which increases blood pressure [11].

Some studies were done in animals which reported the effect of nonionic intravascular contrast media administration on blood pressure during CT scan. Harnish et al. performed a study on the rabbit in which it was observed a variation in blood pressure with contrast media injection and no changes in blood pressure with saline injection [12]. The author concludes that systemic blood pressure increases significantly with contrast administration which agrees with the present study. Whereas, a study conducted by Morris et al. in which contrast media was administrated by hand push method in rabbit observed a decrease in systolic blood pressure [4]. This does not agree with the present study in which contrast media was administered by pressure injector. A rise in intracarotid systolic blood pressure was observed in the dog by Saithoh et al., during the interventional procedure while injecting contrast media through the catheter into the carotid artery [13]. These observations are supporting the current studies observation, which states that contrast administration causes an increase in blood pressure.
Table 1: Mean systolic blood pressure before and after the CT scan

\begin{tabular}{ll}
\hline Measurement of Systolic Blood Pressure & Mean \pm SD \\
\hline Before scan & \\
CECT & $116.64 \pm 6.349$ \\
NCECT & $119.59 \pm 5.188$ \\
After scan & \\
CECT & $128.08 \pm 6.489$ \\
NCECT & $120.14 \pm 5.832$ \\
\hline
\end{tabular}

$\mathrm{n}=146$, SD: Standard deviation, CECT: Contrast-enhanced computed tomography, NCECT: Non-contrast-enhanced computed tomography, CT: Computed tomography

Table 2: Mean diastolic blood pressure before and after the CT scan

\begin{tabular}{ll}
\hline Measurement of Diasystolic Blood Pressure & Mean \pm SD \\
\hline Before scan & \\
CECT & $77.33 \pm 6.351$ \\
NCECT & $80.41 \pm 6.495$ \\
After scan & \\
CECT & $87.19 \pm 5.95$ \\
NCECT & $81.51 \pm 7.105$ \\
\hline
\end{tabular}

$\mathrm{n}=146$, SD: Standard deviation, CECT: Contrast-enhanced computed tomography, NCECT: Non-contrast-enhanced computed tomography, CT: Computed tomography

\section{CONCLUSION}

By analyzing the blood pressure variation during the CECT abdomen scan, we concluded that an increase in both systolic and diastolic blood pressures is associated with intravascular administration of non-ionic low-osmolar contrast media. Further studies can be done by involving the measurement of catecholamine immediately after the contrast injection.

\section{ACKNOWLEDGMENT}

We thank the Institutional Research Committee, SOAHS, and Ethical Committee, Kasturba Hospital, Manipal, for their support and permission to undertake this study. We extend our gratitude to the faculties and Head of the Department of Radio-diagnosis and Imaging, Kasturba Medical College, Manipal, for their cooperation during data collection. We also acknowledge the support of the department of statistics for the guidance during the data analysis and interpretation.

\section{AUTHOR'S CONTRIBUTION}

Arathy Mary John and Sushil Yadav designed the study. Arathy Mary John performed data analysis. Arathy Mary John and Sushil Yadav wrote manuscript.

\section{CONFLICTS OF INTEREST}

No conflicts of interest exist with this study.

\section{REFERENCES}

1. Bae KT. Intravenous contrast medium administration and scan timing at CT: Considerations and appoaches. Radiology 2010;256:32-61.

2. Kumar L. Harmacovigilance/reporting adverse drug reactions: An approach to enhance health surveillance and extending market share by minimizing the chances of drug withdrawals. Int J Pharm Pharm Sci 2015;7:1-7.

3. Juchem BC, Dall'Agnol CM. Immidiate Adverse reactions to intravenous iodinated contrast median in computed tomography. Rev Latinoam Enfermagem 2007;15:78-83.

4. Morris TW, Prentice L, Ventura J. A comparison of the systemic responses to rapid intravenous injections of ioxilan, iohexol, and diatrizoate in rabbits. Invest Radiol 1989;24:294-7. 
5. Rossi P, Young IS, Panke WF. Techniques, usefulness, and hazards of arteriography of pheochromocytoma: Review of 99 cases. JAMA 1968;205:547-53.

6. Gold RE, Wisinger BM, Geraci AR, Heinz LM. Hypertensive crisis as a result of adrenal venography in a patient with pheochromocytoma. Radiology 1972;102:579-80.

7. Aspelin P, Stacul F, Thomsen HS, Morcos SK, van der Molen AJ. Effects of iodinated contrast media on blood and endothelium. Eur Radiol 2006;16:1041-9.

8. Cooper MW, Reed P J. Comparison of ionic and non-ionic contrast agents in cardiac catheterization: The effects of ventriculography and coronary arteriography on hemodynamics, electrocardiography, and serum creatinine. Cathet Cardiovasc Diagn 1991;22:267-77.

9. Sachiko N, Yoshito T, Ayako TT, Tetsuya H, Makato A, Keigo E, et al.
Hypertensive crisis due to contrast-enhanced computed tomography in a patient with malignant pheochromocytoma. Jpn J Radiol 2011;29:449-51.

10. Tamuli1 S, Kakati S, Das S, Singh KD, Ghosh SK. Comparative studies of efficacy and effects on oxidative stress of amlodipine and ramipril in the hypertensive patients of north east India. Int J Pharm Pharm Sci 2015;7:118-21.

11. Bald KS, Edwln LW, Robert WA, Henrl LA. Brief communication: Radiographic contrast infusion and catecholamine release in patients with pheochromocytoma. Ann Intern Med 2009;150:27-33.

12. Harnish P, Mukherji M, Kido DK. Physiologic effects of contrast media in the rabbit. Invest Radiol 1987;22:901-4.

13. Saithoh H, Hayakawa K, Nishimura K, Okuno Y, Murayama C, Miyazawa $\mathrm{T}$, et al. Intracarotidnlood pressure changes during contrast medium injection. AJNR Am J Neuroradiol 1996;17:51-4. 